\title{
Gustatory Dysfunction: A Highly Specific and Smell-Independent Symptom of COVID-19
}

\author{
Luigi Angelo Vaira ${ }^{1}$ (D) Jerome R. Lechien ${ }^{2,3} \cdot$ Giovanni Salzano $^{4} \cdot$ \\ Francesco Antonio Salzano ${ }^{5}$ Fabio Maglitto $^{4} \cdot$ Sven Saussez $^{2,3} \cdot$ Giacomo De Riu $^{1}$
}

Received: 30 August 2020/Accepted: 23 September 2020/Published online: 30 September 2020

(C) Association of Otolaryngologists of India 2020

\begin{abstract}
Chemosensitive dysfunctions are now considered as frequent and early symptoms of coronavirus disease 2019 (COVID-19). In the last few weeks, researchers' greatest efforts have been focusing mainly on the analysis of olfactory disorders, neglecting taste dysfunctions. According to our psychophysical evaluations, it can be inferred that the pathogenesis of taste disorders in COVID19 patients is largely smell-independent. Moreover, isolated gustatory disorders are highly specific of SARS-CoV2 infection. For these reasons, it is essential that gustatory dysfunctions, like olfactory disorders, are included in the COVID-19 guidelines.
\end{abstract}

Keywords COVID-19 - SARS-CoV-2 · Coronavirus · Chemosensitive dysfunction - Olfactory dysfunction . Ageusia $\cdot$ Taste $\cdot$ Gustatory dysfunction $\cdot$ Anosmia

Luigi Angelo Vaira

luigi.vaira@gmail.com

1 Maxillofacial Surgery Operative Unit, University Hospital of Sassari, Viale San Pietro 43/B, 07100 Sassari, Italy

2 COVID-19 Task Force of the Young-Otolaryngologists of the International Federations of Oto-Rhino-Laryngological Societies (YO-IFOS), Mons, Belgium

3 Department of Human and Experimental Oncology, Faculty of Medicine UMONS Research Institute for Health Sciences and Technology, University of Mons (UMons), Mons, Belgium

4 Maxillofacial Surgery Unit, University Hospital of Naples "Federico II", Via Pansini 5, 80131 Naples, Italy

5 Otolaryngology Operative Unit, Department of Medicine, Surgery and Dentistry, "Scuola Medica Salernitana", University of Salerno, 84081 Baronissi, Salerno, Italy

\section{Introduction}

Chemosensitive dysfunctions are now considered as frequent and early symptoms of coronavirus disease 2019 (COVID-19) in Europe and America, affecting 60-80\% of patients [1-4]. In the last few weeks, researchers' greatest efforts have been focusing mainly on the analysis of olfactory disorders, neglecting taste dysfunctions, for two main reasons. According to a first hypothesis, the olfactory pathway could represent a way of access to the central nervous system for the coronavirus [5]. Therefore, olfactory dysfunctions could represent a sign of neuroinvasion, while ageusia and hypogeusia would not have any possible interesting pathogenetic implication. Secondly, the gustatory disturbances have generally been classified as a consequence of the retronasal olfactory loss and for this reason they have been often overlooked [6].

After the outbreak of the severe acute respiratory syndrome coronavirus 2 (SARS-CoV-2) pandemic in Italy, we immediately began evaluating patients with psychophysical tests [7-10]. In this way, we were able to obtain a large amount of objective data that allowed us to evaluate the relationships between olfactory and gustatory disorders in COVID-19 patients.

\section{Methodology}

A standardized and validated test, which investigates the ability to perceive four primary tastes (sweet, salty, sour and bitter) was used to evaluate the gustatory function in patients with confirmed diagnosis of SARS-CoV-2 infection [7-10]. Four solutions, one for each primary taste, were prepared as follows [11]: 
- Salted solution: $30 \mathrm{~g}$ of table salt were added to $1 \mathrm{~L}$ of deionized water.

- Sweet solution: $30 \mathrm{~g}$ of refined sugar were dissolved in $1 \mathrm{~L}$ of deionized water.

- Sour solution: $90 \mathrm{~mL}$ of commercial $100 \%$ lemon juice added to $1 \mathrm{~L}$ of deionized water.

- Bitter solution: unsweetened decaffeinated coffee.

Deionized water was used as control. During the trial, $1 \mathrm{ml}$ of each solution was dropped onto the center of the patient tongue. Taste score ranged from 0 to 4 allowing to classify the patients into four categories: Normal (score 4), mild hypogeusia (score 3), moderate hypogeusia (score 2), severe hypogeusia (score 1) and ageusia (score 0). At the same time, all the patients underwent olfactory assessment with the Connecticut Chemosensory Clinical Research Center orthonasal olfaction test and a self-administered psychophysical olfactory test recently validated by our group [7-10].

\section{Results}

With this methodology, we obtained objective assessments of the olfactory and gustatory functions of 556 patients, many of which have been prospectively assessed several times. In the 801 evaluations performed, isolated taste disturbances were found in $9.4 \%$ of cases. Considering also subjects with combined dysfunctions, a total of $51.1 \%$ of patients reported gustatory disorders. Moreover, in 17.9\% of patients with combined dysfunctions, taste disorders were more severe than the olfactory ones. This frequency is probably underestimated as $74.4 \%$ of these evaluations were performed over 10 days after the symptom onset. As a matter of fact, in most patients the gustatory dysfunction tends to regress completely within 10 days, while the recovery of the olfactory disorder takes longer [7, 9, 12]. Taking into consideration only the 205 assessments that took place within 10 days after clinical onset, patients with gustatory disorders rose to $69.2 \%$ with a frequency of isolated disorders of $11.6 \%$. In this early stage subgroup, $26.3 \%$ of patients who presented combined chemosensitive disorders had a gustatory dysfunctions more severe than the olfactory one (Table 1).

\section{Discussion}

The studies currently present in the literature, have not been able to detect these results as they are based exclusively on anamnestic data or on psychophysical evaluation of patients in the late stage of the disease, when the gustatory disorder had already regressed. Only the objective evaluation of the patients during the early stage of the infection permits to appropriately compare the frequency and severity of the two chemosensitive disorders.

According to these results, it can be inferred that the pathogenesis of taste disorders in COVID-19 patients is largely smell-independent. Anosmia is typically associated with viral infections while ageusia could represent a further manifestation, related to the loss of the retronasal olfactory function. The ability of SARS-CoV-2 to induce gustatory disturbances in the absence of olfactory dysfunctions is instead a unique peculiarity for a virus.

The mechanism by which SARS-CoV-2 is capable of causing gustatory disturbances could be twofold. First, the virus uses the angiotensin converting enzyme 2 (ACE2), receptors widely expressed on the taste buds, to infect cells. Ageusia is a well known side effect of ACE2-inhibitors and it is caused by a complex mechanism which involves sodium channel and G-protein-coupled protein present in taste receptors. SARS-CoV-2, binding these receptors, might inactivate them, hindering the conversion of chemical gustatory signals into action potential, consequently precluding the sensory perception of taste [13, 14].

Moreover, SARS-CoV-2 might bind to sialic acid receptors [15]. Sialic acid is a fundamental component of the salivary mucin and protects the glycoproteins that convey gustatory molecules inside the taste pores from premature enzymatic degradation. A reduction of sialic acid in the saliva is associated with an increase in the gustatory threshold. Therefore, SARS-CoV-2 could occupy the binding sites of sialic acid on the taste buds, accelerating the degradation of the gustatory particles [13].

From a diagnostic point of view, isolated gustatory disorders are very important as they are highly specific of SARS-CoV-2 infection. In fact, olfactory dysfunction is also present in $12 \%$ of cases of common flu [16] and, although useful as a screening marker, it can give a significant number of false positives. At the beginning of the epidemic in Italy, an abnormal number of patients turned to our clinic for the detection of sudden onset ageusia, not associated with any other symptoms. Punctually, SARSCoV-2 infection was then diagnosed in these patients following the further onset of fever and cough.

\section{Conclusion}

Otorhinolaryngologists, maxillofacial surgeons, neurologists and dentists should be warned that in patients seeking assistance for the isolated sudden onset of ageusia, suspicion of ongoing coronavirus infection should be considered in differential diagnosis. For these reasons, in our opinion it is essential that gustatory dysfunctions, like olfactory disorders, are included in the COVID-19 guidelines as they 
Table 1 Chemosensitive function psychophysical analysis results

\begin{tabular}{lll}
\hline & Overall (No. 801) & $<10$ days (No. 205) \\
\hline Isolated taste dysfunction & $76(9.4 \%)$ & $23(11.6 \%)$ \\
Combined dysfunction & $334(41.7 \%)$ & $114(57.6 \%)$ \\
Taste $>$ smell & $36(10.8 \%)$ & $30(26.3 \%)$ \\
Taste $=$ smell & $107(32 \%)$ & $51(44.7 \%)$ \\
Taste $<$ smell & $191(57.2 \%)$ & $33(28.9 \%)$ \\
Isolated olfactory dysfunction & $217(27.1 \%)$ & $35(17.7 \%)$ \\
No dysfunction & $174(21.7 \%)$ & $35(17.7 \%)$ \\
\hline
\end{tabular}

are frequent, highly specific and smell-independent symptoms of SARS-CoV-2 infection.

Funding None declared.

\section{Compliance with Ethical Standards}

Conflict of interest The authors declare that they have no conflict of interest.

Ethical Approval The evaluation protocol was approved by an independent ethical committee (No. 378-2020-OSS-AUSLBO).

Informed Consent All patients gave their consent to participate in the study.

\section{References}

1. Spinato G, Fabbris C, Polesel J (2020) Alterations in smell or taste in mildly symptomatic outpatients with SARS-CoV-2 infection. JAMA 323:2089-2090

2. Lechien JR, Ducarme M, Place S (2020) Objective olfactory findings in hospitalized severe COVID-19 patients. Pathogens 9:627

3. Vaira LA, Salzano G, Deiana G, Riu De G (2020) Ageusia and anosmia: common findings in COVID-19 patients. Laryngoscope 130:1787

4. Vaira LA, Salzano G, Riu De G (2020) The importance of olfactory and gustatory disorders as early symptoms of coronavirus disease (COVID-19). Br J Oral Maxillofac Surg 58:615-616

5. Vavougios GD (2020) Potentially irreversible olfactory and gustatory impairments in COVID-19. Indolent versus fulminant SARS-CoV-2 neuroinfection. Brain Behav Immun 87:107-108

6. Whitcroft KL, Hummel $\mathrm{T}$ (2020) Olfactory dysfunction in COVID-19. Diagnosis and management. JAMA 323:2512-2514
7. Vaira LA, Hopkins C, Petrocelli M (2020) Do olfactory and gustatory psychophysical scores have a prognostic value in COVID-19 patients? A prospective study of 106 patients. J Otolaryngol Head Neck Surg 49:56

8. Vaira LA, Salzano G, Petrocelli M, Deiana G, Salzano FA, Riu De G (2020) Validation of a self-administered olfactory and gustatory test for the remotely evaluation of COVID-19 patients in home quarantine. Head Neck 42:1570-1576

9. Vaira LA, Hopkins C, Salzano G (2020) Olfactory and gustatory function impairment in COVID-19 patients: an Italian objective multicenter-study. Head Neck 42:1560-1569

10. Petrocelli M, Ruggiero F, Baietti AM (2020) Remote psychophysical evaluation of olfactory and gustatory functions in early-stage coronavirus disease 2019 patients: the Bologna experience of 300 cases. J Laryngol Otol 134:571-576

11. Massarelli O, Vaira LA, Biglio A (2018) Sensory recovery of myomucosal flap oral cavity reconstructions Head Neck 40:467-474

12. Vaira LA, Hopkins C, Petrocelli M (2020) Smell and taste recovery in coronavirus disease 2019 patients: a 60-day objective and prospective study. J Laryngol Otol 134:703-709

13. Vaira LA, Salzano G, Fois AF, Piombino P, Riu De G (2020) Potential pathogenesis of ageusia and anosmia in COVID-19 patients. Int Forum Allergy Rhinol 10:1103-1104

14. Lechien JR, Radulesco T, Calvo-Henriquez C (2020) ACE2 \& TMPRSS2 expressions in head \& neck tissues: a systematic review. Head Neck Pathol. https://doi.org/10.1007/s12105020-01212-5

15. Milanetti M, Miotto M, Di Rienzo L, Monti M, Gosti G, Ruocco G (2020) In-Silico evidence for two receptors based strategy of SARS-CoV-2. ArXiv. https://doi.org/2003.11107

16. Lechien JR, Hopkins C, Saussez S (2020) Letter to the editor about the Beltràn-Corbellini et al. Publication: "acute-onset smell and taste disordes in the context of Covid-19: a pilot multicenter PCR-based case-control study". Eur J Neurol. https://doi.org/ 10.1111/ene. 14357

Publisher's Note Springer Nature remains neutral with regard to jurisdictional claims in published maps and institutional affiliations. 